\title{
Investigation of the Risk of Infection of Urinary Schistosomiasis at Mahem and Galilea Communities in the Greater Accra Region of Ghana
}

\author{
I. F. Aboagye* and D. Edoh \\ Department of Zoology, University of Ghana, Legon, Accra, Ghana \\ *Corresponding author; E-mail: iaboagye@ug.edu.gh
}

\begin{abstract}
Urinary schistosomiasis is of great public health importance in developing countries. It has adverse economic and health implications on residents living in endemic areas. Various factors including human behaviour are known to play key role in the transmission of the disease. The knowledge of the levels of risk of infection of urinary schistosomiasis and people's perception will be an important tool in its control. The study determined the prevalence of urinary schistosomiasis and the risk of infection in some communities near the Weija lake in the Ga District. It assessed the knowledge base of the subjects on the disease and its impact on transmission. Data were collected on demographic variables, some behavioural activities in water bodies, knowledge base on the disease and sanitary facilities. Urine samples were analysed using the centrifugation technique. The percentage prevalence for Mahem and Galilea were 58\% and $49 \%$, respectively. The difference in prevalence was insignificant; $0.09(-0.04,0.21 ; P<0.426)$. Bloody urine was associated with high risk of infection; OR of 4.55 (2.82, 7.36); $P<0.001$. Subjects with primary level of education and invariably below 26 years of age had about two times the risk of infection; OR of $2.12(1.13,3.97) ; P<0.02$. The communities had 52\% prevalence of urinary schistosomiasis. Frequent contacts and use of the infested lake were associated with infection. Educational intervention alone may not be effective in the control of the disease. The use of an integrated approach should be given favourable consideration.
\end{abstract}

\section{Introduction}

Urinary schistosomiasis is a disease of great public health importance and remains an important cause of morbidity and mortality globally, particularly in the developing world. After malaria, schistosomiasis is the second most prevalent tropical parasitic disease, and is a leading cause of morbidity in endemic areas of Africa, Asia and South America (WHO, 1995). In some parts of Africa the onset of haematuria due to urinary schistosomiasis is so common in adolescent boys that it is seen as normal (Desowitz, 1981). It is estimated that approximately 200-300 million people are suffering from schistosomiasis in the world (Nash et al., 1982; Savioli, 1990).

It is known that Schistosoma haematobium, which causes urinary schistosomiasis, is the predominant human schistosome species in Ghana, and it is widely distributed in the country (McCullough \& Ali, 1965; Onori et al., 1963). In Ghana, recent studies indicate that the disease is of great public health importance. A study by Aryeetey et al. (2000) revealed that prevalence of Schistosoma haematobium infection in some communities drained by the Densu river ranged between $54.8 \%$ and $60.0 \%$.

Various factors, including human behaviour, are known to be important in the transmission of the disease. Human behavioural activities, especially fishing, fetching of water, swimming, washing, and others, play a role in the transmission of the disease (Wagatsuma et al., 2003; Useh \& Ejezie, 1999). Identifying the levels of risk of infection of urinary schistosomiasis and knowing the general perception of people about the disease will be an important tool in its control.

\section{Study area}

\section{Materials and methods}

A cross-sectional study was carried out in two communities (Galilea and Mahem) in the Ga District of the Greater Accra Region of Ghana to investigate the problem of urinary schistosomiasis. These communities are in the western part of the District and close to the Weija lake, believed to be infested with schistosome parasites. The inhabitants of these communities rely on the Weija lake for various activities of which fishing and bathing are no exception. Although there are some other sources of water for the communities, water from the lake is put to a variety of uses. Mahem is more rural compared with Galilea. Fig. 1 gives geographical description of the study area and its immediate environment. 


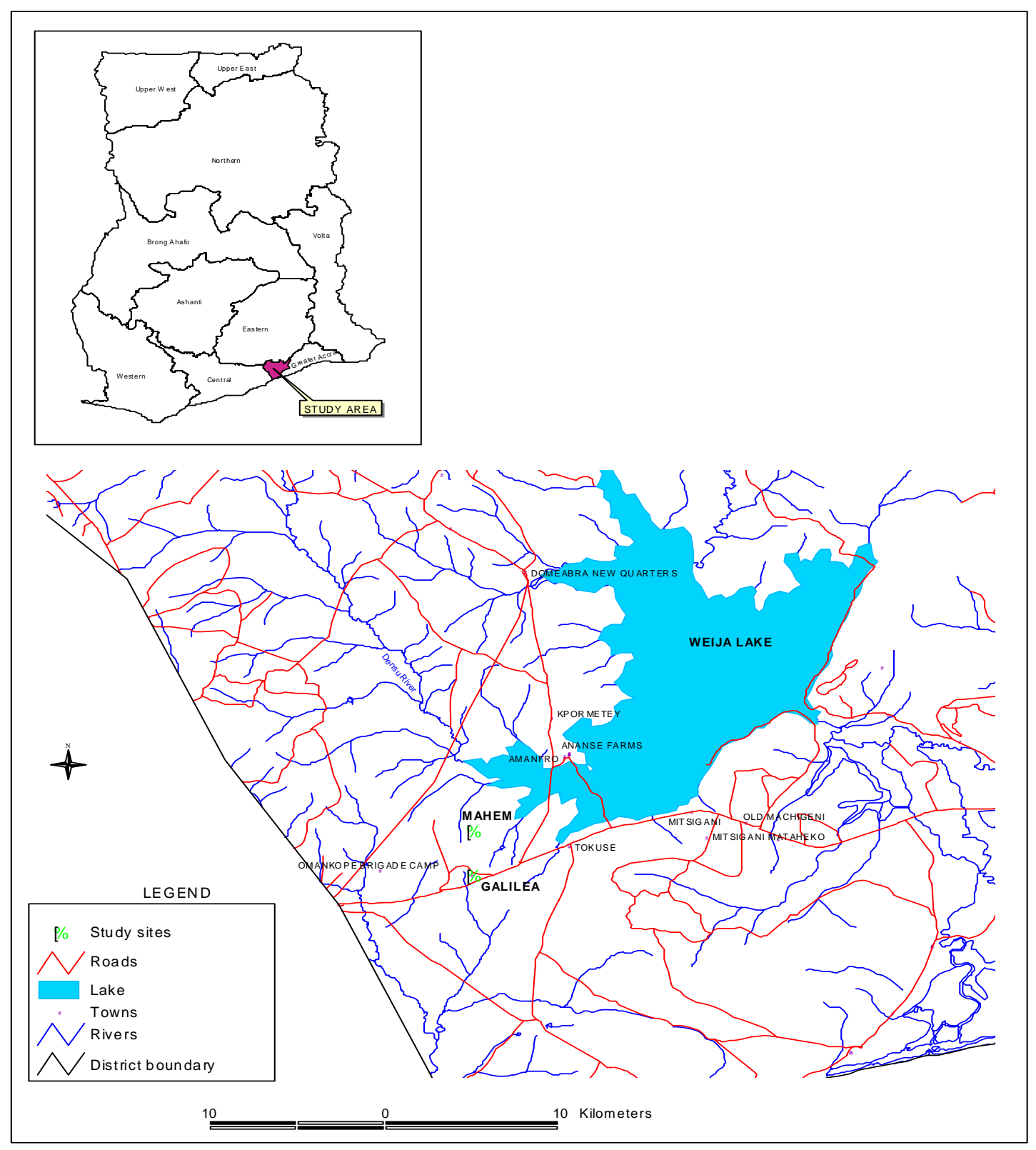

Fig. 1. Geographical description of the study area

\section{Data collection and analysis}

The study was carried out between October and April. Questionnaire was designed and used as the data collection tool. It covered common demographic variables, some behavioural activities in water bodies, knowledge base on the disease system and sanitary facilities available in the communities. To reduce selection bias 309 individuals, aged 5 years or more and of both sexes with different educational background and occupation from the communities, were interviewed at random. The questionnaire was structured in a more detailed and specific manner and the content explained in a language best understood by respondents as a way of reducing measurement error. Prior to this, the purpose of the study was explained to them. All those who declined to participate fully or agreed to participate partially were left out of the study.

\section{Parasitological analysis}

10-ml urine sample was collected from each subject. All samples were collected between 10 a.m. and 2 p.m. and kept at a temperature of $4-6{ }^{\circ} \mathrm{C}$ till they were sent to the laboratory for analysis. Each fresh urine sample was tested for the presence of blood as soon as possible using CYBOW ${ }^{\mathrm{TM}}$ reagent strips, as hematuria and self-reported blood in urine have been shown to be simple and useful indicators for urinary schistosomiasis (Anosike et al., 2001; Lengeler et al., 2002). Individual urine samples were poured into test tubes and centrifuged at a speed of 1000 r.p.m. for $10 \mathrm{~min}$. The sediment for each sample was prepared on a slide for observation under a microscope. Thorough observation of all prepared slides was done under a micro-scope at $\times 40$ objective lens. The number of schistosome eggs present in each preparation was counted 
and recorded using the following grading; + [1-5 schistosome eggs per slide (eps)], ++ (1-10 schistosome eps), +++ (1-20 schistosome eps) and ++++ (more than 20 schistosome eps).

The data were analysed using the logistic regression model in Statistical Package for Social Sciences (SPSS) to investigate the association between the risk of urinary schistosomiasis infection and other variables. The $\mathrm{Z}$ test was used to compare the prevalence of the disease in both communities and to assess the statistical significance of the observed difference in prevalence.

\section{Results}

The infection of Schistosoma haematobium was found in the communities studied. In all, 160 (52\%) subjects out of 309 were found to have urinary schistosomiasis. The percentage prevalence for Mahem and Galilea were $58 \%$ and $49 \%$, respectively. The observed difference in prevalence of the disease in the communities was not significant; $0.09(-0.04,0.21 ; P<0.426)$. The risk of infection of the disease for demographic variables and blood urine expressed as odds ratio (OR) is shown in Table 1. Subjects with blood urine had high risk of infection of 4.55 (2.82, 7.36); $P<0.001$ when compared with those without blood in urine. Fishermen had infection risk of $2.05(0.75,5.55) ; P<0.160$ when compared with the unemployed.

TABLE 1

Association of the risk of urinary schistosomiasis infection with sex, age, occupation, level of education and bloody urine. Values are numbers (percentages)

\begin{tabular}{|c|c|c|c|c|c|}
\hline Variable & $\begin{array}{l}\text { Infected } \\
\text { subjects }\end{array}$ & *NSES & $\begin{array}{l}P \text { value } \\
\text { (Wald's) }\end{array}$ & $\begin{array}{l}\text { P value } \\
\text { (LRT) }\end{array}$ & $* *$ OR $(95 \% C I)$ \\
\hline \multicolumn{6}{|l|}{ Sex } \\
\hline Male & 79 (50.3) & $83(54.6)$ & & & 1.0 \\
\hline Female & 78 (49.7) & $69(45.4)$ & $<0.451$ & $<0.451$ & $1.19(0.76,1.86)$ \\
\hline Age (years) & & & $<0.515$ & $<0.486$ & \\
\hline$>45$ & $4(2.5)$ & $9(5.9)$ & & & 1.0 \\
\hline $5-15$ & $78(49.7)$ & $70(46.1)$ & $<0.140$ & & $2.51(0.74,8.50)$ \\
\hline $16-25$ & $50(31.8)$ & 44 (28.9) & $<0.140$ & & $2.56(0.74,8.88)$ \\
\hline $26-35$ & $16(10.2)$ & $21(13.8)$ & $<0.433$ & & $1.71(0.45,6.58)$ \\
\hline $36-45$ & $9(5.7)$ & $8(5.3)$ & $<0.230$ & & $2.53(0.56,11.51)$ \\
\hline Occupation & & & $<0.418$ & $<0.390$ & \\
\hline Unemployed & $16(10.2)$ & $18(11.8)$ & & & 1.0 \\
\hline Farmers & $5(3.2)$ & $5(3.3)$ & $<0.870$ & & $1.13(0.27,4.61)$ \\
\hline Artisans & $6(3.8)$ & 12 (7.9) & $<0.343$ & & $0.56(0.17,1.85)$ \\
\hline Traders & 21 13.4) & $24(15.8)$ & $<0.972$ & & $0.98(0.40,2.40)$ \\
\hline Students & 89 (56.7) & 82 (53.9) & $<0.595$ & & $1.22(0.58,2.55)$ \\
\hline Fishermen & $20(12.7)$ & $11(7.2)$ & $<0.160$ & & $2.05(0.75,5.55)$ \\
\hline Level of education & & & $<0.160$ & $<0.152$ & \\
\hline None & $20(12.7)$ & $33(21.7)$ & & & 1.0 \\
\hline Primary & $100(63.7)$ & 78 (51.3) & $<0.020$ & & $2.12(1.13,3.97)$ \\
\hline JSS/Middle & $27(17.2)$ & 33 (21.7) & $<0.435$ & & $1.35(0.64,2.87)$ \\
\hline Sec./SSS & $9(5.7)$ & $7(4.6)$ & $<0.193$ & & $2.12(0.68,6.59)$ \\
\hline Blood urine & & & $<0.001$ & $<0.001$ & \\
\hline No & $55(35)$ & $108(71.1)$ & & & 1.0 \\
\hline Yes & $102(65)$ & 44 (28.9) & $<0.001$ & & $4.55(2.82,7.36)$ \\
\hline
\end{tabular}

* No schistosome egg seen in urine sample

** Odd Ratio (95\% confidence interval)

Table 2 gives statistical description of the association between some water contact variables, source of water and knowledge of source of infection on one hand and infection of the disease on the other. Subjects with primary level of education who spend more time in the infested water and invariably below 26 years of age had risk of $2.12(1.13,3.97) ; P<0.02$. There was increasing strength of association between frequent contact with the water body and infection. Subjects who had $>4$ times contact with the infested water body per week had risk of $1.50(0.76,2.95) ; P<0.242$ in comparison with those who had no activity in the water body. The associations of other variables with risk of infection of the disease are given in Table 2. 
TABLE 2

Associations of the risk of urinary schistosomiasis infection with some water contact variables, source of water and knowledge of source of infection. Values are numbers (percentages)

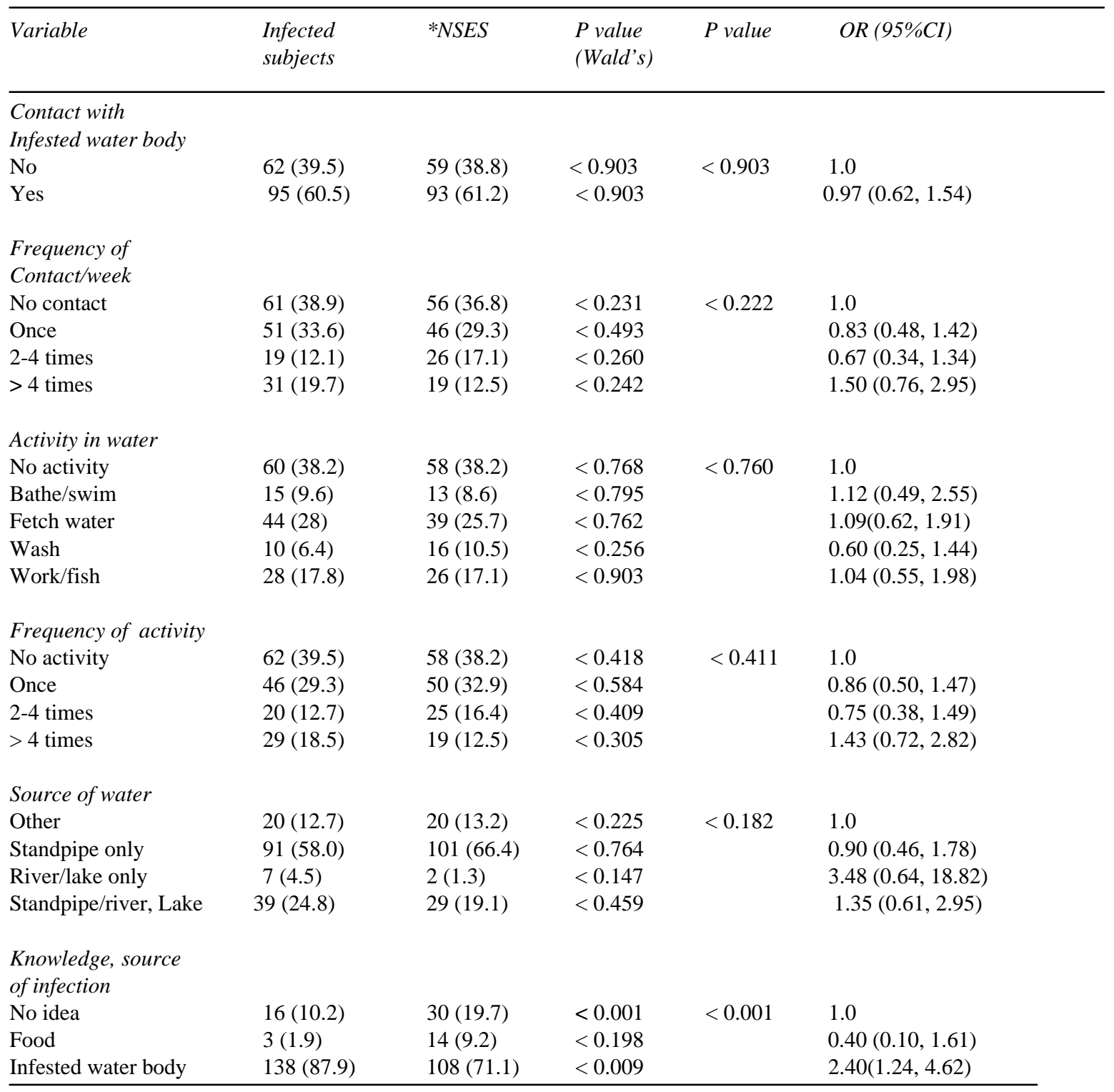

As high as $80 \%$ (246) of the subjects know that an infested water body is the source of infection of the disease. Equal percentage $(33 \%, 102)$ of them attributed infestation of water bodies to either urinating or urinating/defaecating into water bodies. Knowledge of infested water body as source of infection had risk of 2.40 (1.24, 4.62); $P<0.009$.

\section{Discussion}

The study reveals that urinary schisto-somiasis is a disease of great public health importance in the Mahem and Galilea communities in Ghana. It is highly endemic in the communities, with overall prevalence of 52\%, an indication that the problem needs urgent attention. The prevalence of the disease is $9 \%$ higher in Mahem than in Galilea. As revealed by Kloos et al. (1997), intensity of infection and water contact decline relatively slowly with distance from infested water body. The difference could, therefore, be due to proximity of Mahem to the infested water body. However, this difference is not significant; 0.09 (-0.04, 0.21); $P<0.426$, suggesting that the disease is equally important in both communities. Factors such as lack of safe alternative water sources for some residents, water contact behaviour and some types of occupation may cause this high prevalence.

There was highly significant association between blood urine and infection of the disease with blood urine having a risk of $4.55(2.82,7.36) ; P<0.001$. Blood urine is a common symptom of urinary schisto-somiasis. In highly endemic areas, blood urine may, therefore, be useful as an indicator of infection of the disease. 
The risk of infection of fishermen was two times that of the unemployed, $2.05(0.75,5.55) ; P<0.160$, but the $P$-value shows it is not significant. This may be explained by the relatively smaller sample size in that category. However, fishing was found to have stronger association with infection of the disease compared with other occupations and may be due to regular contact with infested water body. It is important to note that $35 \%$ (109) of the subjects admitted taking medication to cure the disease (regularly for some of them and $<6$ months prior to the study for others) on seeing blood urine. This observation may partly explain the results.

Moreover, frequent contact with infested water body was found to be very important. The risk of infection for more than four times contact with infested water body was found to be higher (43\%) than those who do not have contact with the water body. Similar trend was observed with the frequency of activity in infested water body. However, the association was not significant and may be explained by other factors including regular medication.

The results on the relationship between urinary schistosomiasis infection and demographic variables indicate that, overall, there was no significant association between them, although some of the variables were more important than others. Compared to males, females appear to have a risk of $19 \%$ more than that of males i.e. 1.19; $P<0.450$. However, this difference was not significant implying that both males and females are equally vulnerable. All age groups are at risk of infection. The likely reason is contact with infested water bodies.

The young (below 26 years) appear to be at increased risk of infection probably because fetching of water, bathing/swimming in water bodies, among others, which have been identified to be risk factors by some earlier studies (Wagatsuma et al., 2003; Useh \& Ejezie, 1999; Enk et al., 2003; Jeans \& Schwellnus, 1994), are frequently carried out by them. The overall $P$ values for both Wald's test $(P<0.160)$ and likelihood ratio test $(P<0.152)$ indicate that the level of education of subjects has no relationship with infection of the disease. Subjects with primary level of education, who are almost invariably below 26 years of age and spend more time in the infested lake, have about two times risk of infection. This finding may be explained by the fact that children at the primary level of education are attracted to swimming.

The subjects generally have high knowledge of the disease system. This is because earlier interventions have given them the knowledge base. However, there was a strong association between infection of the disease and knowledge of source of infection $(P<0.001)$, suggesting that the subjects do not apply the knowledge or have no alternatives to their water needs. Knowledge of source of infection is, therefore, not protective against urinary schistosomiasis infection; thus, validating the findings of some earlier studies of which those of Wagatsuma et al. (2003) and Sama \& Ratard (1994) are included. This suggests that educational intervention alone cannot be used to address effectively the problem of schistosomiasis control. The use of an integrated approach in urinary schisto-somiasis control should, therefore, be given favourable consideration.

In conclusion, the communities investigated had 52\% prevalence of the disease. Blood urine, frequent contact and use of infested lake water were associated with high risk. Self-medication may have influence on the findings. This is because the drug of choice, Praziquantel, is effective against all schistosome species occurring in man. Metriphonate, a monospecific anti-schistosomal drug, is also effective against Schistosoma haematobium (Davis, 1996). Moreover, very large sample size will provide more precise estimate of effect. Further study is recommended in other communities exposed to the risk of infection of the disease.

\section{Acknowledgement}

The authors wish to thank the Department of Zoology, University of Ghana, for the logistical support. Thanks are also due to Mr Francis Seku, Prosper Buo, Godfred Futagbe and Oheneba Kofi Nti, for the diverse ways in which they contributed to the success of the study. The co-operation of the people in the study area is appreciated.

\section{References}

Anosike J. C., Nwoke B. E. B. and Njoku A. J. (2001). The validity of haematuria in the community diagnosis of urinary schistosomiasis infections. J. Helminthol. 75: 223-225.

Aryeetey M. E., Wagatsuma Y., Yeboah G., Asante M., Mensah G., Nkrumah F. K. and Kojima S. (2000). Urinary schistosomiasis in southern Ghana: 1 . Prevalence and morbidity assessment in three (defined) rural areas drained by the Densu river. Parasit. Int. 49(2): 155-163.

Davis, A. (1996). Schistosomiasis. In Manson’s Tropical Diseases. (C. G. Cook, ed.), pp. 1413-1456. W. B. Saunders Company Ltd, Great Britain.

Desowitz R. S. (1981) Controlling the schistosomiasis at a snail's pace. In New Guinea Tapeworms and Jewish Grandmothers: Tales of Parasites and People. (R. S. Desowitz, ed.) Norton, New York. 
Enk M. J., Amorim A. and Schall V. T. (2003). Acute schistosomiasis outbreak in the metropolitan area of Belo Horizonte, Minas Gerais: alert about the risk of unnoticed transmission increased by growing rural tourism. Mem. Inst. Oswaldo Cruz 98 (6).

Jeans A. K. and Schwellnus M. P. (1994). The risk of schistosomiasis in Zimbabwean triathletes. S. Afr. Med. J. 84(11): 756-8.

Kloos H., Fulford A. C. A., Butterworth A. E., Sturrock R. F., Ouma J. M., Kariuki H. C., Thiongo F. W., Dalton P. R. and Klumpp R. K. (1997). Spatial patterns of human water contact and Schistosoma mansoni. transmission and infection in four rural areas in Machakos District, Kenya. Soc. Sci. Med. 44: 949-968.

Lengeler C., Utzinger J. and Tanner M. (2002). Questionnaires for rapid screening of schistosomiasis in sub-Saharan Africa. Bull Wld Hlth Organ. 80: 235-242.

McCullough F. S. and Ali Y. M. (1965). The distribution and prevalence of Schistosoma haematobium and Schistosoma mansoni in Ghana. Ghana Med. J. 4: 83-84.

Nash T. E., Cheever A. W., Ottesen E. A. and Cook J. A. (1982). Schistosome infections in humans: perspectives and recent findings. Ann. Int. Med. 97: 740-754.

Onori, E., McCullough F. S. and Rosei L. (1963). Schistosomiasis in the Volta region of Ghana. Ann. Trop. Med. Parasit. 57: 59-70.

Sama M. T. and Ratard R. C. (1994). Water contact and schistosomiasis infection in Kumba, south-western Cameroon. Ann. trop. Med. Parasit. 88(6): 629-34.

Savioli L. (1990). Schistosomiasis control programme on Pemba Island, Zanzibar. In Spirit of enterprise: the 1990 Rolex awards. (D. W. Reed, ed.), pp. 18-20. Buri Druck A. G., Bern.

Useh M. F. and Ejezie G. C. (1999). Modification of behaviour and attitude in the control of schistosomiasis. 1. Observations on water-contact patterns and perception of infection. Ann. Trop. Med. Parasit. 93(7): 711-20.

Wagatsuma Y., Aryeetey M. E., Nkrumah F. K., Sack D. A. and Kojima S. (2003). Highly symptom-aware children were heavily infected with urinary schistosomiasis in southern Ghana. Cen. Afr. J. Med. 49(1-2): 16-9.

World Health Organisation (1995). A Picture of Health? WHO, Geneva/UNICEF, New York. 